\title{
UPPER BOUNDS FOR THE $L_{q}$ NORM OF FEKETE POLYNOMIALS ON SUBARCS
}

\author{
TAMÁS ERDÉLYI
}

Abstract. For a prime $p$ the polynomial

$$
f_{p}(z):=\sum_{k=1}^{p-1}\left(\frac{k}{p}\right) z^{k}
$$

where the coefficients are Legendre symbols, is called the $p$-th Fekete polynomial. In this paper the size of the Fekete polynomials on subarcs is studied. We prove essentially sharp bounds for the average value of $\left|f_{p}(z)\right|^{q}, 0<q<\infty$, on subarcs of the unit circle even in the cases when the subarc is rather small. Our upper bounds are matching with the lower bounds proved in a preceding paper for the $L_{0}$ norm of the Fekete polynomials on subarcs of the unit circle.

\section{INTRODUCTION}

Finding polynomials in the class

$$
\mathcal{L}_{n}:=\left\{Q: Q(z)=\sum_{k=0}^{n} a_{k} z^{k}, \quad a_{k} \in\{-1,1\}\right\}
$$

with small uniform norm on the unit circle raised the interest of many authors. Observe that the uniform norm of any polynomial in $\mathcal{L}_{n}$ on the unit circle is always at least $(n+1)^{1 / 2}$ since the $L_{2}$ norm of any such polynomial is $(2 \pi(n+1))^{1 / 2}$ by the Parseval formula. It is difficult to exhibit a polynomial $Q \in \mathcal{L}_{n}$ with uniform norm at most $C(n+1)^{1 / 2}$ for all $n$ with an absolute constant $C$. An example known was found by H.S. Shapiro [Sh-51] and W. Rudin [Ru-59]. A nice account of this and related problems were given by Littlewood in [Li-69, pages 25-32]. For a prime number $p$ the $p$-th Fekete polynomial is defined as

$$
f_{p}(z):=\sum_{k=1}^{p-1}\left(\frac{k}{p}\right) z^{k}
$$

Key words and phrases. $L_{q}$ norm on subarcs of the unit circle, polynomials with constrained coefficients, Fekete polynomials, Littlewood polynomials.

2000 Mathematics Subject Classifications. 11C08, 41A17, 30C15, 52A40 
where

$$
\left(\frac{k}{p}\right)=\left\{\begin{array}{l}
1, \quad \text { if } x^{2} \equiv k(\bmod p) \text { has a nonzero solution } \\
0, \quad \text { if } p \text { divides } k \\
-1, \quad \text { otherwise }
\end{array}\right.
$$

is the usual Legendre symbol. Since $f_{p}$ has constant coefficient 0 , it is not a Littlewood polynomial, but $g_{p}(z):=f_{p}(z) / z$ is a Littlewood polynomial, and has the same modulus as $f_{p}$ has on the unit circle. Fekete polynomials are examined in detail in [B-02] and [CG-00].

Let $\alpha<\beta$ be real numbers. The Mahler measure $M_{0}(Q,[\alpha, \beta])$ is defined for bounded measurable functions $Q\left(e^{i t}\right)$ defined on $[\alpha, \beta]$ as

$$
M_{0}(Q,[\alpha, \beta]):=\exp \left(\frac{1}{\beta-\alpha} \int_{\alpha}^{\beta} \log \left|Q\left(e^{i t}\right)\right| d t\right) .
$$

It is well known that

$$
M_{0}(Q,[\alpha, \beta])=\lim _{q \rightarrow 0+} M_{q}(Q,[\alpha, \beta])
$$

where

$$
M_{q}(Q,[\alpha, \beta]):=\left(\frac{1}{\beta-\alpha} \int_{\alpha}^{\beta}\left|Q\left(e^{i t}\right)\right|^{q} d t\right)^{1 / q}, \quad q>0 .
$$

It is a simple consequence of the Jensen formula that

$$
M_{0}(Q,[0,2 \pi])=|c| \prod_{k=1}^{n} \max \left\{1,\left|z_{k}\right|\right\}
$$

for every polynomial of the form

$$
Q(z)=c \prod_{k=1}^{n}\left(z-z_{k}\right), \quad c, z_{k} \in \mathbb{C} .
$$

In [M-80] Montgomery proved that there is an absolute constant $c$ such that

$$
\max _{t \in[0,2 \pi]}\left|f_{p}\left(e^{i t}\right)\right| \leq c p^{1 / 2} \log p
$$

for all primes $p$. In fact a closer look of his argument shows that combining Lemma 1.1 due to Gauss and the upper bound for the Lebesgue constant for trigonometric interpolation on equidistant nodes given in [CR-76, Theorem 1] implies that

$$
\max _{t \in[0,2 \pi]}\left|f_{p}\left(e^{i t}\right)\right| \leq p^{1 / 2}\left(\frac{5}{3}+\frac{2}{\pi} \log \frac{p-1}{2}\right) .
$$

Montgomery [Mo-80] also showed that the lower bound

$$
\frac{2}{\pi} p^{1 / 2} \log \log p<\max _{t \in[0,2 \pi]}\left|f_{p}\left(e^{i t}\right)\right|
$$


holds for all sufficiently large primes $p$. No better upper and lower bounds than those of Montgomery are known even today.

In [EL-07] we proved that for every $\varepsilon>0$ there is a constant $c_{\varepsilon}$ such that

$$
M_{0}\left(f_{p},[0,2 \pi]\right) \geq\left(\frac{1}{2}-\varepsilon\right) p^{1 / 2}
$$

for all primes $p \geq c_{\varepsilon}$. One of the key lemmas in the proof of the above theorem formulates a remarkable property of the Fekete polynomials. A simple proof of it is given in [B-02, pp. 37-38].

Lemma 1.1 (Gauss). We have

$$
f_{p}\left(z_{p}^{j}\right)=\varepsilon_{p}\left(\frac{j}{p}\right) p^{1 / 2}, \quad j=1,2, \ldots, p-1,
$$

and $f_{p}(1)=0$, where

$$
z_{p}:=\exp \left(\frac{2 \pi i}{p}\right)
$$

is the first $p$-th root of unity, and $\varepsilon_{p} \in\{-1,1,-i, i\}$.

The choice of $\varepsilon_{p}$ is more subtle. This is also a result of Gauss, see [Hua-82].

Lemma 1.2 (Gauss). In Lemma 1.1 we have

$$
\varepsilon_{p}= \begin{cases}1, & \text { if } p \equiv 1(\bmod 4) \\ i, & \text { if } p \equiv 3(\bmod 4)\end{cases}
$$

In [Er-11] the author extended (1.1) to subarcs of the unit circle. Namely it is proved that there is an absolute constant $c_{1}>0$ such that

$$
M_{0}\left(f_{p},[\alpha, \beta]\right) \geq c_{1} p^{1 / 2}
$$

for all prime numbers $p$ and for all $\alpha, \beta \in \mathbb{R}$ such that $(\log p)^{3 / 2} p^{-1 / 2} \leq \beta-\alpha \leq 2 \pi$.

\section{New Results}

We give an upper bound for the average value of $\left|f_{p}(z)\right|^{q}$ over any subarc $I$ of the unit circle, valid for all sufficiently large primes $p$ and exponents $q>0$.

Theorem 2.1. There is a constant $c_{2}(q, \varepsilon)$ depending only on $q>0$ and $\varepsilon>0$ such that

$$
\left(\frac{1}{|I|} \int_{I}\left|f_{p}(z)\right|^{q}|d z|\right)^{1 / q} \leq c_{2}(q, \varepsilon) p^{1 / 2},
$$

for every subarc $I$ of the unit circle with length $|I| \geq 2 p^{-1 / 2+\varepsilon}$.

We remark that together with the result from [Er-10] mentioned at the end of the Introduction, Theorem 2.1 shows that there is an absolute constant $c_{1}>0$ and a constant $c_{2}(q, \varepsilon)>0$ depending only on $q>0$ and $\varepsilon>0$ such that

$$
c_{1} p^{1 / 2} \leq\left(\frac{1}{|I|} \int_{I}\left|f_{p}(z)\right|^{q}|d z|\right)^{1 / q} \leq c_{2}(q, \varepsilon) p^{1 / 2}
$$

for every subarc $I$ of the unit circle with $|I| \geq 2 p^{-1 / 2+\varepsilon} \geq(\log p)^{3 / 2} p^{-1 / 2}$. 
Theorem 2.2. For every sufficiently large prime $p$ and for every $8 \pi p^{-1 / 8} \leq s \leq 2 \pi$ there is a closed subset $E:=E_{p, s}$ of the unit circle with linear measure $|E|=$ s such that

$$
\frac{1}{|E|} \int_{E}\left|f_{p}(z)\right||d z| \geq c_{3} p^{1 / 2} \log \log (1 / s)
$$

with an absolute constant $c_{3}>0$.

\section{ProOFs}

Our proof of Theorem 2.1 is a combination of Lemma 1.1 due to Gauss, a well-known direct theorem of approximation due to Jackson, and the Marcinkiewicz-Zygmund inequality [MZ-37], [Zy-77, Theorem 7.5, Chapter X]. The Marcinkiewicz-Zygmund inequality asserts that there is a constant $c_{4}(q)$ depending only on $q$ such that

$$
c_{4}(q)^{-1} \frac{1}{n} \sum_{j=1}^{n}\left|P\left(z_{n}^{j}\right)\right|^{q} \leq \int_{0}^{2 \pi}\left|P\left(e^{i t}\right)\right|^{q} d t \leq c_{4}(q) \frac{1}{n} \sum_{j=1}^{n}\left|P\left(z_{n}^{j}\right)\right|^{q}
$$

for any polynomial $P$ of degree at most $n-1$ and for any $1<q<\infty$, where

$$
z_{n}:=\exp \left(\frac{2 \pi i}{n}\right)
$$

is the first $n$-th root of unity.

Proof of Theorem 2.1. It is well known that

$$
\left(\frac{1}{|I|} \int_{I}\left|f_{p}(z)\right|^{q}|d z|\right)^{1 / q}
$$

is an increasing function of $q$ on $(0, \infty)$. So it is sufficient to prove the theorem only for $q>\varepsilon^{-1}>2$. Let $q>1$, we will use $q \geq \varepsilon^{-1}>2$ only at the end of the proof. Without loss of generality we may assume that $|I| \leq 2 \pi / 3$. We introduce the truncated Fekete polynomials $f_{p, m}$ by

$$
f_{p, m}(z):=\sum_{k=1}^{p-(m+1)}\left(\frac{k}{p}\right) z^{k}
$$

with $m:=\left\lfloor p^{1 / 2}\right\rfloor$. Then $f_{p, m}$ is a polynomial of degree $p-(m+1)$. Let $I=\left\{e^{i t}: t \in[a, b]\right\}$ and let $3 I:=\left\{e^{i t}: t \in[2 a-b, 2 b-a]\right\}$ be the arc centered at the midpoint of $I$ with arclength $3|I|$. We define the piecewise linear function $L_{I}$ on on $[2 a-b, 2 a-b+2 \pi]$ first by

$$
L_{I}(t):=\left\{\begin{array}{l}
\frac{1, \quad \text { if } t \in[a, b],}{b-a}, \quad \text { if } t \in[2 a-b, a], \\
\frac{(2 b-a)-t}{b-a}, \quad \text { if } t \in[b, 2 b-a], \\
0, \quad \text { if } t \in[2 b-a, 2 a-b+2 \pi],
\end{array}\right.
$$


and then we extend it as a periodic function with period $2 \pi$ defined on $\mathbb{R}$. By a wellknown direct theorem of approximation (see [DL-93, p. 205], for example) there is a real trigonometric polynomial $T_{m}$ of degree at most $m / 2$ such that

$$
\max _{t \in \mathbb{R}}\left|L_{I}(t)-T_{m}(t)\right| \leq \frac{c_{5}}{m|I|} \leq \frac{1}{2}
$$

with an absolute constant $c_{5}>0$. Without loss of generality we may assume that $T_{m}(t) \geq 0$ for every $t \in \mathbb{R}$, hence $T_{m}(t)=\left|Q_{m}\left(e^{i t}\right)\right|$ with an appropriate algebraic polynomial $Q_{m}$ of degree at most $m$. Note that $\frac{1}{2} \leq\left|Q_{m}(z)\right| \leq \frac{3}{2}$ for every $z=e^{i t} \in I$. Observe that

$$
\left|f_{p}(z)-f_{p, m}(z)\right| \leq m, \quad z=e^{i t}, \quad t \in \mathbb{R} .
$$

Using Lemma 1.1 and (3.2) we can deduce that

$$
\left|f_{p, m}\left(z_{p}^{j}\right)\right| \leq\left|f_{p}\left(z_{p}^{j}\right)\right|+\left|f_{p, m}\left(z_{p}^{j}\right)-f_{p}\left(z_{p}^{j}\right)\right| \leq p^{1 / 2}+m, \quad j=1,2, \ldots, p
$$

Combining the inequality

$$
|a+b|^{q} \leq 2^{q-1}\left(|a|^{q}+|b|^{q}\right), \quad a, b \in \mathbb{C}, \quad q \in[1, \infty)
$$

with (3.2), and then recalling that $\frac{1}{2} \leq\left|Q_{m}(z)\right|$ for all $z=e^{i t} \in I$, we obtain

$$
\begin{aligned}
\int_{I}\left|f_{p}(z)\right|^{q}|d z| & \leq \int_{I} 2^{q-1}\left(\left|f_{p, m}(z)\right|^{q}+\left|f_{p}(z)-f_{p, m}(z)\right|^{q}\right)|d z| \\
& =2^{q-1} \int_{I}\left|f_{p, m}(z)\right|^{q}|d z|+2^{q-1} \int_{I}\left|f_{p}(z)-f_{p, m}(z)\right|^{q}|d z| \\
& \leq 2^{q-1} \int_{I}\left|f_{p, m}(z)\right|^{q}|d z|+2^{q-1} m^{q}|I| \\
& \leq 2^{q-1} 2^{q} \int_{I}\left|\left(f_{p, m} Q_{m}\right)(z)\right|^{q}|d z|+2^{q-1} m^{q}|I| .
\end{aligned}
$$

Applying the Marcinkiewicz-Zygmund inequality to the polynomial

$$
P:=f_{p, m} Q_{m}
$$

of degree at most $p-1$, then using (3.3), we obtain

$$
\begin{aligned}
\int_{I}\left|\left(f_{p, m} Q_{m}\right)(z)\right|^{q}|d z| & \leq c_{4}(q) \frac{1}{p} \sum_{j=1}^{p}\left|\left(f_{p, m} Q_{m}\right)\left(z_{p}^{j}\right)\right|^{q} \\
& \leq c_{4}(q)\left(p^{1 / 2}+m\right)^{q} \frac{1}{p} \sum_{j=1}^{p}\left|Q_{m}\left(z_{p}^{j}\right)\right|^{q} .
\end{aligned}
$$


Observe that (3.1) implies that

$$
\begin{gathered}
\left|Q_{m}\left(z_{p}^{j}\right)\right|^{q} \leq 2^{q}, \quad z_{p}^{j} \in 3 I, \\
\left|Q_{m}\left(z_{p}^{j}\right)\right|^{q} \leq\left(\frac{c_{5}}{m|I|}\right)^{q}, \quad z_{p}^{j} \notin 3 I,
\end{gathered}
$$

and there are at most $\frac{3 p|I|}{2 \pi}+1$ values of $j=1,2, \ldots, p$ for which $z_{p}^{j} \in 3 I$. Hence

$$
\begin{aligned}
\frac{1}{p} \sum_{j=1}^{p}\left|Q_{m}\left(z_{p}^{j}\right)\right|^{q} & \leq \frac{1}{p}\left(2^{q}\left(\frac{3 p|I|}{2 \pi}+1\right)+\left(\frac{c_{5}}{m|I|}\right)^{q} p\right) \\
& \leq\left(2^{q}\left(\frac{3|I|}{2 \pi}+\frac{1}{p}\right)+\left(2 c_{5}\right)^{q}|I|\right) \\
& \leq c_{6}(q)|I|
\end{aligned}
$$

with a constant $c_{6}(q)$ depending only on $q$, whenever

$$
\left(\frac{c_{5}}{m|I|}\right)^{q} \leq\left(2 c_{5}\right)^{q}|I|,
$$

that is, whenever

$$
\frac{1}{m} \leq 2 p^{-1 / 2} \leq 2|I|^{1+1 / q}
$$

Combining (3.4), (3.5), and (3.6), and recalling that $m \leq p^{1 / 2}$, we conclude

$$
\begin{aligned}
\frac{1}{|I|} \int_{I}\left|f_{p}(z)\right|^{q}|d z| & \leq \frac{4^{q}}{|I|}\left(\int_{I}\left|\left(f_{p, m} Q_{m}\right)(z)\right|^{q}|d z|\right)+2^{q} m^{q} \\
& \leq \frac{4^{q}}{|I|} c_{4}(q)\left(p^{1 / 2}+m\right)^{q} \frac{1}{p}\left(\sum_{j=1}^{p}\left|Q_{m}\left(z_{p}^{j}\right)\right|^{q}\right)+2^{q} m^{q} \\
& \leq 4^{q} c_{4}(q) 2^{q} p^{q / 2} c_{6}(q)+2^{q} m^{q} \\
& \leq c_{7}(q) p^{q / 2}
\end{aligned}
$$

with a constant $c_{7}(q)$ depending only on $q$, whenever

$$
\frac{1}{m} \leq 2 p^{-1 / 2} \leq 2|I|^{1+1 / q}
$$

So the theorem is proved for all $q>0$ satisfying

$$
\frac{-1 / 2}{1+1 / q} \leq-\frac{1}{2}+\varepsilon
$$

hence for all $q>\varepsilon^{-1}>2$, with a constant $c_{2}(q, \varepsilon)$ depending only on $q$ and $\varepsilon$.

To prove Theorem 2.2 we follow [Mo-80]. Let $e(t)=\exp (2 \pi i t)$. Our first lemma is stated as Lemma 1 and proved in [Mo-80]. 
Lemma 3.1. Let $\chi$ be a primitive character $(\bmod q), q>1$. Then

$$
\sum_{m=0}^{q-1} \chi(m) e(m \alpha)=\tau(\chi) q^{-1} e\left(\frac{1}{2} q \alpha\right)(\sin (\pi q \alpha)) T(\alpha, \bar{\chi})
$$

where $\tau(\chi)$ is the Gauss sum

$$
\tau(\chi)=\sum_{n=1}^{q} \chi(n) e\left(\frac{n}{q}\right)
$$

and

$$
T(\alpha, \chi)=\sum_{a=1}^{q} \chi(a) \cot \left(\pi\left(\alpha-\frac{a}{q}\right)\right) .
$$

Note that in the case if

$$
\chi(n)=\left(\frac{n}{p}\right)
$$

is the quadratic character, then Lemma 1.1 implies $\tau(\chi)=\varepsilon_{p} p^{1 / 2}$, and the content of Lemma 3.1 is just the identity obtained by expressing the Fekete polynomial $f_{p}$ by the Lagrange interpolation formula associated with the $p$-th root of unity. In fact, in the proof of Theorem 2.2 we will need Lemma 3.1 above only in the case when $\chi$ is the quadratic character.

Our second lemma is stated as Lemma 2 and proved in [Mo-80].

Lemma 3.2. Let $p$ be a prime. For $k \geq 1$ let $a_{1}, a_{2}, \ldots, a_{k}$ be integers, distinct $(\bmod p)$, and put $f(x)=\prod_{j=1}^{k}\left(x-a_{j}\right)$. Then

$$
\left|\sum_{n=1}^{p}\left(\frac{f(n)}{p}\right)\right| \leq(k-1) p^{1 / 2} .
$$

Montgomery writes "This is a consequence of Weil's Riemann Hypothesis for the zeta function of a curve over a finite field: see Weil [We-45], [We-49]. The derivation of the particular bound above is given by Burgess ([Bu-57]; 22$). "$

Proof of Theorem 2.2. We rely heavily on Montgomery's beautiful line of proof in [Mo-80] to connect the two lemmas above to the proof of the theorem. Let $T(\alpha):=T(\alpha, \chi)$ with

$$
\chi(h)=\left(\frac{h}{p}\right) .
$$

It follows from Lemma 1.1 that $|\tau(\chi)|=p^{1 / 2}$ and hence Lemma 3.1 implies that

$$
\left|f_{p}\left(e\left(\frac{2 n+\delta}{2 p}\right)\right)\right| \geq \frac{1}{\sqrt{2}} p^{-1 / 2}\left|T\left(\frac{2 n+\delta}{2 p}\right)\right|
$$


for every $n=1,2, \ldots, p$ and $\delta \in\left[\frac{1}{2}, \frac{3}{2}\right]$. We define

$$
W(n):=W_{H}(n):=\prod_{h=1}^{H}\left(1-\left(\frac{n+h}{p}\right)\right) \prod_{h=0}^{H}\left(1+\left(\frac{n-h}{p}\right)\right),
$$

and compute the size of the weighted sum

$$
\sum_{n=1}^{p} T\left(\frac{2 n+\delta}{2 p}\right) W(n)
$$

for $\delta \in\left[\frac{1}{2}, \frac{3}{2}\right]$. By multiplying the product (3.8) out, we have

$$
W(n)=1+\sum_{f} \varepsilon_{f}\left(\frac{f(n)}{p}\right), \quad \varepsilon_{f} \in\{-1,1\}
$$

where $f$ runs through $2^{2 H+1}-1$ polynomials of the sort considered in Lemma 3.2. Hence, using Lemma 3.2 we can deduce that

$$
\sum_{n=1}^{p} W(n)=p+O\left(H 2^{2 H} p^{1 / 2}\right)
$$

Similarly,

$$
\sum_{n=1}^{p} W(n)\left(\frac{n-a}{p}\right)=c(a) p+O\left(H 2^{2 H} p^{1 / 2}\right)
$$

where $c(a)=1$ if $0 \leq a \leq H, c(a)=0$ if $H<a<p-H$, and $c(a)=-1$ if $p-H \leq a<p$. We have

$$
\begin{aligned}
& \sum_{n=1}^{p} T\left(\frac{2 n+\delta}{2 p}\right) W(n) \\
= & \sum_{n=1}^{p} \sum_{a=1}^{p}\left(\frac{a}{p}\right) \cot \left(\pi\left(\frac{2 n+\delta}{2 p}-\frac{a}{p}\right)\right) W(n) \\
= & \sum_{a=1}^{p} \sum_{n=1}^{p}\left(\frac{n-a}{p}\right) W(n) \cot \left(\pi\left(\frac{2 a+\delta}{2 p}\right)\right) \\
= & \sum_{a=1}^{H}+\sum_{a=p-H}^{p}+\sum_{a=H+1}^{p-H-1}
\end{aligned}
$$

for every $\delta \in\left[\frac{1}{2}, \frac{3}{2}\right]$. Using (3.10) and the facts that

$$
\cot x=-\cot (\pi-x)=\left\{\begin{array}{c}
x^{-1}+O(x), \quad \text { if } x \in(0, \pi / 2], \\
-(\pi-x)^{-1}+O(\pi-x), \quad \text { if } x \in[\pi / 2, \pi),
\end{array}\right.
$$


and

$$
\sum_{a=H+1}^{p-H-1} \cot \left(\pi\left(\frac{2 a+\delta}{2 p}\right)\right)=O\left(\sum_{a=H+1}^{p-H-1} \frac{p}{a}\right)=O(p \log p)
$$

we obtain

$$
\begin{aligned}
& \sum_{a=1}^{H}+\sum_{a=p-H}^{p}+\sum_{a=H+1}^{p-H-1} \\
= & \frac{4 p^{2}}{\pi} \sum_{a=1}^{H} \frac{1}{2 a-1}+O\left(p^{2}\right)+O\left(H 2^{2 H} p^{1 / 2} p \log p\right) \\
= & \frac{2}{\pi} p^{2} \log H+O\left(p^{2}\right)+O\left(H 2^{2 H} p^{1 / 2} p \log p\right) \\
= & \frac{2}{\pi} p^{2} \log H+O\left(p^{2}\right)
\end{aligned}
$$

whenever $\delta \in\left[\frac{1}{2}, \frac{3}{2}\right]$ and $2 \leq H \leq \frac{1}{8} \log p$. Combining (3.11) and (3.12), we conclude

$$
\sum_{n=1}^{p} T\left(\frac{2 n+\delta}{2 p}\right) W(n)=\frac{2}{\pi} p^{2} \log H+O\left(p^{2}\right)
$$

whenever $\delta \in\left[\frac{1}{2}, \frac{3}{2}\right]$ and $2 \leq H \leq \frac{1}{8} \log p$.

Now let $A:=A_{p, H}$ be the union of all intervals

$$
\left[\frac{2 n+\frac{1}{2}}{2 p}, \frac{2 n+\frac{3}{2}}{2 p}\right]
$$

with $W(n):=W_{H}(n) \neq 0, n=1,2, \ldots, p$. We define $B=B_{p, H}:=\{e(t): t \in A\}$. Note that

$$
W(n) \in\left\{2^{2 H}, 2^{2 H+1}, 0\right\}, \quad n=1,2, \ldots, p .
$$

This, together with (3.9), implies that the linear measure of $B$ is

$$
|B| \leq \frac{p}{2^{2 H}} \frac{2 \pi}{2 p}+O\left(H p^{-1 / 2}\right)=\left(\pi+O\left(p^{-1 / 4} \log p\right)\right) 2^{-2 H}
$$

whenever $2 \leq H \leq \frac{1}{8} \log p$. Also $|B| \leq 2 \pi 2^{-2 H}$ for all sufficiently large primes $p$ and for 
all integers $2 \leq H \leq \frac{1}{8} \log p$. Using (3.7) we obtain

$$
\begin{aligned}
\int_{B}\left|f_{p}(z)\right||d z| & =2 \pi \int_{A}\left|f_{p}(e(t))\right| d t \\
& =\frac{\pi}{p} \sum_{\substack{n=1 \\
W(n) \neq 0}}^{p} \int_{1 / 2}^{3 / 2}\left|f_{p}\left(e\left(\frac{2 n+\delta}{2 p}\right)\right)\right| d \delta \\
& \geq \frac{\pi}{p} \frac{1}{\sqrt{2}} p^{-1 / 2} \sum_{\substack{n=0 \\
W(n) \neq 0}}^{p} \int_{1 / 2}^{3 / 2}\left|T\left(\frac{2 n+\delta}{2 p}\right)\right| d \delta \\
& \geq \frac{\pi}{\sqrt{2}} p^{-3 / 2} \int_{1 / 2}^{3 / 2}\left(\sum_{\substack{n=1 \\
W(n) \neq 0}}^{p} T\left(\frac{2 n+\delta}{2 p}\right)\right) d \delta .
\end{aligned}
$$

Using (3.14) and (3.13) we can continue as

$$
\begin{aligned}
& \frac{\pi}{\sqrt{2}} p^{-3 / 2} \int_{1 / 2}^{3 / 2}\left(\sum_{\substack{n=1 \\
W(n) \neq 0}}^{p} T\left(\frac{2 n+\delta}{2 p}\right)\right) d \delta \\
\geq & \frac{\pi}{\sqrt{2}} p^{-3 / 2} 2^{-(2 H+1)} \int_{1 / 2}^{3 / 2}\left(\sum_{\substack{n=1 \\
W(n) \neq 0}}^{p} T\left(\frac{2 n+\delta}{2 p}\right) W(n)\right) d \delta \\
\geq & \frac{\pi}{\sqrt{2}} p^{-3 / 2} 2^{-(2 H+1)}\left(\frac{2}{\pi} p^{2} \log H+O\left(p^{2}\right)\right) \\
\geq & \frac{\pi}{\sqrt{2}} 2^{-(2 H+1)}\left(\frac{2}{\pi} p^{1 / 2} \log H+O\left(p^{1 / 2}\right)\right) .
\end{aligned}
$$

Thus (3.16) and (3.17) imply

$$
\int_{B}\left|f_{p}(z)\right||d z| \geq \frac{\pi}{\sqrt{2}} 2^{-(2 H+1)}\left(\frac{2}{\pi} p^{1 / 2} \log H+O\left(p^{1 / 2}\right)\right) .
$$

Now let $8 \pi p^{-1 / 8} \leq s \leq 2 \pi$ be fixed. Without loss of generality we may assume that $s \leq 1$. Let $H \geq 2$ be the (only) integer such that

$$
s / 4<2 \pi 2^{-2 H} \leq s .
$$

Then

$$
H \leq \frac{\log p}{16 \log 2} \leq \frac{1}{8} \log p
$$

As $\left|B_{p, H}\right| \leq 2 \pi 2^{-2 H}$ for all sufficiently large primes $p$ and for all integers $2 \leq H \leq \frac{1}{8} \log p$, there is a closed subset $E:=E_{p, s}$ of the unit circle with linear measure $s$ containing $B:=B_{p, H}$. Then (3.18) and (3.19) imply that

$$
\frac{1}{s} \int_{E}\left|f_{p}(z)\right||d z| \geq c\left(p^{1 / 2} \log \log (1 / s)+O\left(p^{1 / 2}\right)\right)
$$

with an absolute constant $c>0$. 


\section{REFERENCES}

B-02. P. Borwein, Computational Excursions in Analysis and Number Theory, Springer, New York, 2002.

Bu-57. D.A. Burgess, The distribution of quadratic residues and non-residues, Mathematika 4 (1957), $106-112$.

CR-76. E.W. Cheney and T.J Rivlin, A note on some Lebesgue constants, Rocky Mountain J. Math. 6 (1976), 435-439.

CG-00. B. Conrey, A. Granville, B. Poonen, and K. Soundararajan, Zeros of Fekete polynomials, Ann. Inst. Fourier (Grenoble) 50 (2000), 865-884.

DL-93. R.A. DeVore and G.G. Lorentz, Constructive Approximation, Springer-Verlag, Berlin, 1993.

Er-11. T Erdélyi, Sieve-type lower bounds for the Mahler measure of polynomials on subarcs, Computational Methods and Function Theory 11 (2011), 213-228.

EL-07. T. Erdélyi and D. Lubinsky, Large sieve inequalities via subharmonic methods and the Mahler measure of Fekete polynomials, Canad. J. Math. 59 (2007), 730-741.

Hua-82. L.K. Hua, Introduction to Number Theory, Springer-Verlag, New York-Berlin, yr 1982.

Li-68. J.E. Littlewood, Some problems in real and complex analysis, Heath (Lexington), 1968.

MZ-37. J. Marcinkiewicz and A. Zygmund, Mean values of trigonometric polynomials, Fund. Math. 28 (1937), 131-166.

Mo-80. H.L. Montgomery, An exponential polynomial formed with the Legendre symbol, Acta Arith. $\mathbf{3 7}$ (1980), 375-380.

Ru-59. W. Rudin, Some theorems on Fourier coefficients, Proc. Amer. Math. Soc. 10 (1959), 855-859.

Sh-51. H.S. Shapiro, Extremal Problems for Polynomials and Power Series (Master's thesis), MIT, 1951.

We-45. A. Weil, Sur les courbes algébriques et les variétés qui s'en déduisent, Actualités Sci. Ind. 1041 = Publ. Inst. Math. Univ. Strasbourg 7 (1945). Hermann et Cie, Paris, 1948.

We-45. A. Weil, Numbers of solutions of equations in finite fields, Bull. Amer. Math. Soc. 55 (1949), 497-508.

Zy-77. A. Zygmund, Trigonometric Series, vols. I, II, Cambridge University Press, Cambridge, 1977.

Department of Mathematics, Texas A\&M University, College Station, Texas 77843

E-mail address: terdelyi@math.tamu.edu 